\title{
HEAT CONDUCTION THROUGH A THIN, POORLY CONDUCTIVE LAYER*
}

BY

L. E. BOBISUD

University of Idaho, Moscow

I. Introduction. Many applied heat-transfer problems involve a thin layer of poor conductivity, such as an oxide layer on the surface of a metal. Heat conduction in a bar capped with a thin oxide coating was considered in [1] for boundary conditions corresponding to specified temperatures maintained at the ends; the effect of the thin layer was, to first approximation, that of replacing the specified-temperature boundary condition with a radiation condition. Here we consider a related model in which a thin sheet of poor conductivity occurs between two conducting materials and seek an asymptotic representation as the thickness and conductivity of this sheet go to zero. In view of the results of [1], we anticipate a change in the form of the connection condition as a reflection of the singularity of the perturbation.

We carry out a detailed analysis for a one-dimensional problem in order to keep the exposition simple. Consider, then, the problem

$$
\begin{aligned}
& u_{t}=c_{u}^{2} u_{x x}, \quad u(x, 0)=h(x) \quad(-1-\varepsilon \leq x \leq-\varepsilon), \\
& v_{t}=\sigma^{2} v_{x x}, \quad v(x, 0)=h(x) \quad(-\varepsilon<x<\varepsilon), \\
& w_{t}=c_{w}^{2} w_{x x}, \quad w(x, 0)=h(x) \quad(\varepsilon \leq x \leq 1+\varepsilon), \\
& u(-1-\varepsilon, t)=u_{0}(t), \quad w(1+\varepsilon, t)=w_{0}(t) \quad(t \geq 0) .
\end{aligned}
$$

Here $\sigma^{2}$ is the coefficient of thermal diffusivity of the poor conductor, which occupies the region $-\varepsilon<x<\varepsilon$; subscripts $u$ and $w$ denote quantities pertaining to the regions $-1-\varepsilon \leq x \leq-\varepsilon$ and $\varepsilon \leq x \leq 1+\varepsilon$, respectively. We require also continuity of temperature:

$$
u(-\varepsilon, t)=v(-\varepsilon, t), \quad v(\varepsilon, t)=w(\varepsilon, t) \quad(t \geq 0),
$$

and conservation of energy:

$$
\begin{gathered}
\rho_{u} C_{u} c_{u}^{2} u_{x}(-\varepsilon, t)=\rho_{v} C_{v} \sigma^{2} v_{x}(-\varepsilon, t), \\
\rho_{v} C_{v} \sigma^{2} v_{x}(\varepsilon, t)=\rho_{w} C_{w} c_{w}^{2} w_{x}(\varepsilon, t) \quad(t \geq 0),
\end{gathered}
$$

where $\rho$ is the density and $C$ the specific heat of the material occupying the indicated region. These latter conditions can be rewritten more conveniently as

$$
k_{u} u_{x}(-\varepsilon, t)=\sigma^{2} v_{x}(-\varepsilon, t), \quad \sigma^{2} v_{x}(\varepsilon, t)=k_{w} w_{x}(\varepsilon, t) \quad(t \geq 0) .
$$

\footnotetext{
* Received March 30, 1982.
} 
where

$$
k_{u} \equiv \rho_{u} C_{u} c_{u}^{2} / \rho_{v} C_{v}, \quad k_{w} \equiv \rho_{w} C_{w} c_{w}^{2} / \rho_{v} C_{v}
$$

It is convenient to reformulate the problem (1-3) in terms of a new independent variable $y$ defined by

$$
\begin{array}{rlrl}
y & \equiv x-1+\varepsilon, & & -1-\varepsilon \leq x \leq-\varepsilon \\
& \equiv x / \varepsilon, & & -\varepsilon<x<\varepsilon \\
& \equiv x+1-\varepsilon, & \varepsilon \leq x \leq 1+\varepsilon
\end{array}
$$

let $\hat{u}(y, t)=u(x(y), t)$, etc. Then

$$
\begin{aligned}
& \hat{u}_{t}=c_{u}^{2} \hat{u}_{y y}, \quad \hat{u}(y, 0)=h(y+1-\varepsilon) \quad(-2 \leq y \leq-1), \\
& \hat{v}_{t}=\frac{\sigma^{2}}{\varepsilon^{2}} \hat{v}_{y y}, \quad \hat{v}(y, 0)=h(\varepsilon y) \quad(-1 \leq y \leq 1), \\
& \hat{w}_{t}=c_{w}^{2} \hat{w}_{y y}, \quad \hat{w}(y, 0)=h(y-1+\varepsilon) \quad(1 \leq y \leq 2), \\
& \hat{u}(-2, t)=u_{0}(t), \quad \hat{w}(2, t)=w_{0}(t) \quad(t \geq 0), \\
& \hat{u}(-1, t)=\hat{v}(-1, t), \quad \hat{v}(1, t)=\hat{w}(1, t) \quad(t \geq 0), \\
& k_{u} \hat{u}_{y}(-1, t)=\frac{\sigma^{2}}{\varepsilon} \hat{v}_{y}(-1, t), \quad \frac{\sigma^{2}}{\varepsilon} \hat{v}_{y}(1, t)=k_{w} \hat{w}_{y}(1, t) \quad(t \geq 0) .
\end{aligned}
$$

Our interest lies in determining an asymptotic expression for $u, w$ in the limit as $\varepsilon$ and $\sigma$ tend to zero in some dependent manner. Restricting ourselves to dependencies of the form

$$
\sigma^{2}=\varepsilon^{n} K,
$$

we find as in [1] that only one value of $n$ yields a particularly interesting reduced problem in the limit as $\varepsilon \rightarrow 0$, namely, $n=1$. If $n<1$, the conductivity is great relative to the thickness of the thin sheet; as a result, the limiting problem corresponds to the absence of the poorly conductive sheet altogether. If $n>1$, the sheet is so nonconductive that the zeroth-order approximation corresponds to two materials thermally isolated from one another at their junction $x=0$. For the case $n=1$, however, there is a more interesting limiting problem; consequently we assume $n=1$ from now on.

Setting $\sigma^{2}=\varepsilon K$ in (4) results in

$$
\begin{aligned}
& \hat{u}_{t}=c_{u}^{2} \hat{u}_{y y}, \quad \hat{u}(y, 0)=h(y+1-\varepsilon) \quad(-2 \leq y \leq-1), \\
& \varepsilon \hat{v}_{t}=K \hat{v}_{y y}, \quad \hat{v}(y, 0)=h(\varepsilon y) \quad(-1<y<1), \\
& \hat{w}_{t}=c_{w}^{2} \hat{w}_{y y}, \quad \hat{w}(y, 0)=h(y-1+\varepsilon) \quad(1 \leq y \leq 2), \\
& \hat{u}(-2, t)=u_{0}(t), \quad \hat{w}(2, t)=w_{0}(t) \quad(t \geq 0), \\
& \hat{u}(-1, t)=\hat{v}(-1, t), \quad \hat{v}(1, t)=\hat{w}(1, t) \quad(t \geq 0), \\
& k_{u} \hat{u}_{y}(-1, t)=K \hat{v}_{y}(-1, t), \quad K \hat{v}_{y}(1, t)=k_{w} \hat{w}_{y}(1, t) \quad(t \geq 0) .
\end{aligned}
$$

The reduced problem is obtained formally by setting $\varepsilon=0$ in (5); we denote the solution by $\hat{u}^{0}, \hat{v}^{0}, \hat{w}^{0}$. Ignoring for now the initial condition $\hat{v}^{0}(y, 0)=h(0)$, corresponding to a 
possible boundary layer at $t=x=0$, we have that $\hat{v}^{0}(y, t)$ must solve

$$
\begin{gathered}
\hat{v}_{y y}^{0}=0, \quad \hat{v}^{0}(-1, t)=\hat{u}^{0}(-1, t), \quad \hat{v}^{0}(1, t)=\hat{w}^{0}(1, t), \\
K \hat{v}_{y}^{0}(-1, t)=k_{u} \hat{u}_{y}^{0}(-1, t), \quad K \hat{v}_{y}^{0}(1, t)=k_{w} \hat{w}_{y}^{0}(1, t) .
\end{gathered}
$$

From (6) it follows that

$$
\hat{v}^{0}(y, t)=\frac{1}{2}(y+1) \hat{w}^{0}(1, t)-\frac{1}{2}(y-1) \hat{u}^{0}(-1, t) ;
$$

hence (7) becomes

$$
k_{u} \hat{u}_{y}^{0}(-1, t)=k_{w} \hat{w}_{y}^{0}(1, t)=\frac{1}{2} K\left[\hat{w}^{0}(1, t)-\hat{u}^{0}(-1, t)\right] .
$$

The first equality in (8) is a statement of conservation of energy; it asserts that energy is not stored or created at the boundary $x=0$. The final equality relates the energy flow through this junction to the temperature differential across it. The constant $K$ is given in physical quantities by $K=\sigma^{2} / \varepsilon$-thermal diffusivity per thickness.

Returning $(5,8)$ to the original variables $x, t$, we have shown formally that the reduced problem is

$$
\begin{aligned}
& u_{t}^{0}=c_{u}^{2} u_{x x}^{0}, \quad u^{0}(x, 0)=h(x) \quad(-1 \leq x \leq 0), \\
& w_{t}^{0}=c_{w}^{2} w_{x x}^{0}, \quad w^{0}(x, 0)=h(x) \quad(0 \leq x \leq 1), \\
& u^{0}(-1, t)=u_{0}(t), \quad w^{0}(1, t)=w_{0}(t) \quad(t \geq 0), \\
& k_{u} u_{x}^{0}(0, t)=k_{w} w_{x}^{0}(0, t)=\frac{1}{2} K\left[w^{0}(0, t)-u^{0}(0, t)\right] \quad(t \geq 0) .
\end{aligned}
$$

The change in the form of the coupling equations from continuity of temperature to the temperature jump condition is a manifestation of the 'singularity' of the original smallparameter problem.

The remainder of this paper is devoted to justifying the solution of (9) as the limit of the solution of (1-3). Throughout the following we assume such smoothness of the data as is necessary to justify the manipulations involved; in particular, the assignment of initial and boundary data is assumed continuous. The following two sections contain lemmas that will be used in the final section to prove the validity of the representation (9).

II. Some lemmas. In this section we establish three lemmas used in proving the validity of the asymptotic representation obtained later. The first of these lemmas is the basic maximum principle for the perturbed problem.

Lemma 1. Let $u, v, w$ satisfy

$$
\begin{array}{cll}
u_{t}=c_{u}^{2} u_{y y}, & u(y, 0) \leq 0 & (-2 \leq y \leq-1), \\
\varepsilon v_{t}=K v_{y y}, \quad v(y, 0) \leq 0 & (-1 \leq y \leq 1), \\
w_{t}=c_{w}^{2} w_{y y}, \quad w(y, 0) \leq 0 & (1 \leq y \leq 2), \\
u(-2, t) \leq 0, & w(2, t) \leq 0, \\
u(-1, t)=v(-1, t), & v(1, t)=w(1, t), \\
k_{u} u_{y}(-1, t)=K v_{y}(-1, t), & K v_{y}(1, t)=k_{w} w_{y}(1, t)
\end{array}
$$

for $0 \leq t \leq T$. Then $u \leq 0, v \leq 0, w \leq 0$ for $|y| \leq 2,0 \leq t \leq T$. 
Proof. Set

$M \equiv \max \left(\max _{0 \leqslant t \leqslant T,-2 \leqslant y \leqslant-1} u(y, t), \max _{0 \leqslant t \leqslant T,-1 \leqslant y \leqslant 1} v(y, t), \max _{0 \leqslant t \leqslant T, 1 \leqslant y \leqslant 2} w(y, t)\right)$, and suppose $M>0$. By the maximum principle applied separately to the regions $-2 \leq y \leq-1,-1 \leq y \leq 1,1 \leq y \leq 2$, we have that $M$ must be assumed along either $y=-1$ or $y=1$ for $t>0$. Suppose without loss of generality that $M$ is achieved at $(1, \bar{t})$. If $\bar{t}<T$, then by the second maximum principle [2, p. 170] we have that $w_{y}(1, \bar{t})<0$, $v_{y}(1, \bar{t})>0$, a contradiction to $K v_{y}(1, \bar{t})=k_{w} w_{y}(1, \bar{t})$. If $\bar{t}=T$, we must have $w_{y}(1, T) \leq 0$, $v_{y}(1, T) \geq 0$, implying that $w_{y}(1, T)=v_{y}(1, T)=0$. Now $w(1, T)=M$ is the maximum of $w$ over $\{(y, t): 1 \leq y \leq 2,0 \leq t \leq T\}$. Set

$$
\begin{aligned}
\phi(t) & \equiv w(1, t), & & 0 \leq t \leq T \\
& \equiv M-t+T, & & T<t \leq T+M,
\end{aligned}
$$

and let $\theta(y, t)$ be the solution of the problem

$$
\begin{aligned}
& \theta_{t}=c_{w}^{2} \theta_{y y}, \quad \theta(y, 0)=w(y, 0) \leq 0 \quad(1 \leq y \leq 2), \\
& \theta(2, t)=w(2, t) \leq 0, \quad \theta(1, t)=\phi(t) \quad(0 \leq t \leq T+M) .
\end{aligned}
$$

Then $\theta$ has its maximum of $M$ at $(1, T)$, and by the second maximum principle, $\theta_{y}(1, T)<0$. But $\theta \equiv w$ for $t \leq T$, so we have $w_{y}(1, T)<0$, a contradiction.

LEMma 2. Let, for some $i \geq 0$,

$$
\begin{array}{ccc}
U_{t}=c_{u}^{2} U_{y y}, & U(y, 0)=O\left(\varepsilon^{i}\right) & (-2 \leq y \leq-1), \\
\varepsilon V_{t}=K V_{y y}, & V(y, 0)=O\left(\varepsilon^{i}\right) & (-1 \leq y \leq 1), \\
W_{t}=c_{w}^{2} W_{y y}, & W(y, 0)=O\left(\varepsilon^{i}\right) & (1 \leq y \leq 2), \\
U(-2, t)=0, & W(2, t)=0 \\
U(-1, t)=V(-1, t), & V(1, t)=W(1, t) \\
k_{u} U_{y}(-1, t)=K V_{y}(-1, t), & K V_{y}(1, t)=k_{w} W_{y}(1, t)
\end{array}
$$

for $0 \leq t \leq T$, where $O\left(\varepsilon^{i}\right)$ holds uniformly in $y$ and $t \in[0, T]$. Then $U, V, W=O\left(\varepsilon^{i}\right)$ uniformly in $y$ and $t \in[0, T]$.

Proof. Let $M$ be such that each term denoted $O\left(\varepsilon^{i}\right)$ is bounded in magnitude by $M \varepsilon^{i}$ uniformly in $y$ and $t \in[0, T]$. It suffices to show that each of $U, V$, and $W$ is bounded above by $M \varepsilon^{i}$, as the corresponding lower bound follows in the same manner. Set $u=$ $U-M \varepsilon^{i}, v=V-M \varepsilon^{i}, w=W-M \varepsilon^{i}$; then $u, v, w$ satisfy Lemma 1 . Therefore $u, v, w \leq 0$, as desired.

Lemma 3. Let (10) and

$$
\begin{aligned}
& U_{t}=c_{u}^{2} U_{y y}, \quad U(y, 0)=0 \quad(-2 \leq y \leq-1), \\
& \varepsilon V_{t}=K V_{y y}+O\left(\varepsilon^{2}\right), \quad V(y, 0)=0 \quad(-1 \leq y \leq 1), \\
& W_{t}=c_{w}^{2} W_{y y}, \quad W(y, 0)=0 \quad(1 \leq y \leq 2)
\end{aligned}
$$

hold, where $O\left(\varepsilon^{2}\right)$ is uniform in $y \in[-1,1]$ and $t \in[0, t]$. Then $U, V$, and $W=O(\varepsilon)$ uniformly in $y$ and $t \in[0, T]$.

Proof. Let the term denoted $O\left(\varepsilon^{2}\right)$ in the hypothesis be $\varepsilon^{2} g(y, t, \varepsilon)$, where $g$ is bound- 
ed. Let $u(y, t, \varepsilon ; \tau), v(y, t, \varepsilon, \tau), w(y, t, \varepsilon ; \tau)$ solve for $t \geq \tau$

$$
\begin{aligned}
& u_{t}=c_{u}^{2} u_{y y}, \quad u(y, \tau, \varepsilon ; \tau)=0 \quad(-2 \leq y \leq-1), \\
& \varepsilon v_{t}=K v_{y y}, \quad v(y, \tau, \varepsilon ; \tau)=g(y, \tau, \varepsilon) \quad(-1 \leq y \leq 1), \\
& w_{t}=c_{w}^{2} w_{y y}, \quad w(y, \tau, \varepsilon ; \tau)=0 \quad(1 \leq y \leq 2), \\
& u(-2, t)=w(2, t)=0, \quad u(-1, t)=v(-1, t), \quad v(1, t)=w(1, t), \\
& k_{u} u_{y}(-1, t)=K v_{y}(-1, t), \quad K v_{y}(1, t)=k_{w} w_{y}(1, t) .
\end{aligned}
$$

It follows from Lemma 2 that $u, v, w$ are bounded uniformly in $y, t, \varepsilon, \tau$ as $\varepsilon \rightarrow 0$. Since an easy calculation shows that

$$
U=\varepsilon \int_{0}^{t} u(\cdot ; \tau) d \tau, \quad V=\varepsilon \int_{0}^{t} v(\cdot ; \tau) d \tau, \quad W=\varepsilon \int_{0}^{t} w(\cdot ; \tau) d \tau,
$$

the lemma follows.

III. Properties of the reduced problem. The form of the reduced problem is

$$
\begin{array}{ccc}
u_{t}=c_{u}^{2} u_{x x}, & u(x, 0)=h(x) & (-1 \leq x \leq 0), \\
w_{t}=c_{w}^{2} w_{x x}, & w(x, 0)=h(x) & (0 \leq x \leq 1), \\
u(-1, t)=u_{0}(t), & w(1, t)=w_{0}(t) & (t \geq 0), \\
k_{u} u_{x}(0, t)+\frac{1}{2} K\{u(0, t)-w(0, t)\}=f(t) & (t \geq 0), \\
k_{w} w_{x}(0, t)+\frac{1}{2} K\{u(0, t)-w(0, t)\}=g(t) & (t \geq 0) .
\end{array}
$$

We show first that this problem possesses a unique solution for suitable data; for this we do not assume that $h$ is continuous at 0 .

Uniqueness is an immediate consequence of the following maximum principle.

Lemma 4. Consider the problem (11-15) above with $h \leq 0, u_{0} \leq 0, w_{0} \leq 0, f=0, g=0$. For any $T>0$ define

$$
\phi=\max \left\{\max _{0 \leqslant t \leqslant T,-1 \leqslant x \leqslant 0} u(x, t), \max _{0 \leqslant t \leqslant T, 0 \leqslant x \leqslant 1} w(x, t)\right\} .
$$

Then $\phi \leq 0$.

Proof. Suppose on the contrary that $\phi>0$. By separate applications of the classical maximum principle in the two regions $-1 \leq x \leq 0$ and $0 \leq x \leq 1$, we see that $\phi$ must be achieved along $x=0$, say at $(0, \bar{t})$. As in the proof of Lemma 1 , we can assume that $0<\bar{t}<T$. Suppose that $\phi=u(0, \bar{t})$. By the second maximum principle, $u_{x}(0, \bar{t})>0$; from (14) with $f \equiv 0$ it follows that $\phi=u(0, \bar{t})<w(0, \bar{t})$, a contradiction. A similar argument eliminates the case $\phi=w(0, \bar{t})$, and the lemma is proved.

We turn now to existence of solutions. Let

$$
G(x, t ; \xi, \tau)=\frac{1}{2 \pi^{1 / 2}(t-\tau)^{1 / 2}}\left[\exp \left\{-\frac{(x-\xi)^{2}}{4 c_{w}^{2}(t-\tau)}\right\}-\exp \left\{-\frac{(x+\xi)^{2}}{4 c_{w}^{2}(t-\tau)}\right\}\right]
$$

be the Green's function for the half plane $x>0$; then $\mathscr{G}(x, t ; \xi, \tau) \equiv G(1-x, t ; 1-\xi, \tau)$ is the Green's function for the region $x<1$. We shall use $\mathscr{G}$ to produce integral equations for $W(t) \equiv w(0, t)$ and $U(t) \equiv u(0, t)$; once $w(0, t)$ and $u(0, t)$ are obtained, $w(x, t)$ and $u(x, t)$ 
can be found as the solutions of standard boundary-value problems. To this end we integrate Green's identity

$$
\frac{\partial}{\partial \xi}\left(\mathscr{G} \frac{\partial w}{\partial \xi}-w \frac{\partial \mathscr{G}}{\partial \xi}\right)-\frac{\partial}{\partial \tau}(\mathscr{G} w)=0
$$

over $0<\xi<1,0<\tau<t$ to get

$$
\begin{aligned}
w(x, t)= & \int_{0}^{t} G(1-x, t ; 1, \tau)\left\{\frac{K}{2 k_{w}}[u(0, \tau)-w(0, \tau)]-\frac{1}{k_{w}} g(\tau)\right\} d \tau \\
& +\int_{0}^{t} G_{\xi}(1-x, t ; 0, \tau) w_{0}(\tau) d \tau-\int_{0}^{t} G_{\xi}(1-x, t ; 1, \tau) w(0, \tau) d \tau \\
& +\int_{0}^{t} G(1-x, t ; 1-\xi, 0) h(\xi) d \xi
\end{aligned}
$$

where we have used the initial-boundary conditions $(11-13,15)$. Taking the limit as $x \rightarrow 0+$ and using known properties of the double-layer heat potential [3, p. 99], we get that

$$
\begin{aligned}
W(t)= & \frac{K}{k_{w}} \int_{0}^{t} G(1, t ; 1, \tau) U(\tau) d \tau \\
& -\int_{0}^{t}\left\{2 G_{\xi}(1, t ; 1, \tau)+\frac{K}{k_{w}} G(1, t ; 1, \tau)\right\} W(\tau) d \tau \\
& -\frac{2}{k_{w}} \int_{0}^{t} G(1, t ; 1, \tau) g(\tau) d \tau+2 \int_{0}^{t} G_{\xi}(1, t ; 0, \tau) w_{0}(\tau) d \tau \\
& +2 \int_{0}^{1} G(1, t ; 1-\xi, 0) h(\xi) d \xi .
\end{aligned}
$$

A similar analysis shows that $U(t)$ satisfies

$$
\begin{aligned}
U(t)= & \frac{K}{k_{u}} \int_{0}^{t} \bar{G}(1, t ; 1, \tau) W(\tau) d \tau \\
& -\int_{0}^{t}\left\{2 \bar{G}_{\xi}(1, t ; 1, \tau)+\frac{K}{k_{u}} \bar{G}(1, t ; 1, \tau)\right\} U(\tau) d \tau \\
& +\frac{2}{k_{u}} \int_{0}^{t} \bar{G}(1, t ; 1, \tau) g(\tau) d \tau+2 \int_{0}^{t} \bar{G}_{\xi}(1, t ; 0, \tau) w_{0}(\tau) d \tau \\
& +2 \int_{-1}^{0} \bar{G}(1, t ; 1+\xi, 0) h(\xi) d \xi
\end{aligned}
$$

where $\bar{G}$ differs from $G$ only in having $c_{u}$ in place of $c_{w}$. Standard arguments show that if $U, W$ satisfy the above pair of integral equations and $u, w$ solve

$$
\begin{aligned}
& u_{t}=c_{u}^{2} u_{x x}, \quad u(x, 0)=h(x) \quad(-1 \leq x \leq 0), \\
& u(-1, t)=u_{0}(t), \quad u(0, t)=U(t) \quad(t \geq 0), \\
& w_{t}=c_{w}^{2} w_{x x}, \quad w(x, 0)=h(x) \quad(0 \leq x \leq 1), \\
& w(1, t)=w_{0}(t), \quad w(0, t)=W(t) \quad(t \geq 0),
\end{aligned}
$$


then $u, w$ solve (11-15). Hence it suffices to show that the integral equations $(16,17)$ possess a solution.

Observe that

$$
\begin{aligned}
& G_{\xi}(1, t ; 0, \tau)=\frac{1}{2 \pi^{1 / 2} c_{w}^{2}(t-\tau)^{3 / 2}} e^{-1 / 4 c_{w}^{2}(t-\tau)}, \\
& G_{\xi}(1, t ; 1, \tau)=\frac{1}{2 \pi^{1 / 2} c_{w}^{2}(t-\tau)^{3 / 2}} e^{-1 / c_{w}^{2}(t-\tau)}
\end{aligned}
$$

are bounded for $t \geq \tau$ because $z^{\alpha} e^{-z}$ is bounded on $0 \leq z$. Also

$$
G(1, t ; 1, \tau)=\frac{1}{2 \pi^{1 / 2}(t-\tau)^{1 / 2}}\left[1-e^{-1 / w^{2}(t-\tau)}\right],
$$

which has an integrable singularity at $t=\tau$. The final integral in (16) can be recast as

$$
2 \int_{0}^{1} \frac{1}{2 \pi^{1 / 2} t^{1 / 2}} e^{-\xi^{2} / 4 c w^{2} t} h(\xi) d \xi-2 \int_{0}^{1} \frac{1}{2 \pi^{1 / 2} t^{1 / 2}} e^{-(2-\xi) 2 / 4 c w^{2} t} h(\xi) d \xi .
$$

Now the first of these integrals is the solution, evaluated at $x=0$, of the Cauchy problem

$$
\begin{aligned}
& v_{t}=c_{w}^{2} v_{x x}, \quad v(x, 0)=0, \quad x<0 \\
& =h(x), \quad 0 \leq x \leq 1 \\
& =0, \quad x>1 \text {; }
\end{aligned}
$$

this integral is therefore a continuous function of $t$ and approaches $\frac{1}{2} h(0+)$ as $t \rightarrow 0+$. Similarly, the second integral is $v(2, t)$ and thus continuous. A similar treatment of (17) shows that $(16,17)$ can be written as

$$
\begin{aligned}
W(t)= & -\int_{0}^{t}\left\{2 G_{\xi}(1, t ; 1, \tau)+\frac{K}{k_{w}} G(1, t ; 1, \tau)\right\} W(\tau) d \tau \\
& +\frac{K}{k_{w}} \int_{0}^{t} G(1, t ; 1, \tau) U(\tau) d \tau+F(t), \\
U(t)= & -\int_{0}^{t}\left\{2 \bar{G}_{\xi}(1, t ; 1, \tau)+\frac{K}{k_{u}} \bar{G}(1, t ; 1, \tau)\right\} U(\tau) d \tau \\
& +\frac{K}{k_{u}} \int_{0}^{t} \bar{G}(1, t ; 1, \tau) W(\tau) d \tau+H(t),
\end{aligned}
$$

where $F$ and $H$ are continuous for $t \geq 0$. From this point standard arguments (either Picard iterations or fixed-point methods) show that this pair of equations has a continuous solution; we leave the details to the reader. The following theorem summarizes these results.

THEOREM 1. Let $h$ be continuous except possibly for a simple discontinuity at 0 , and let $u_{0}, w_{0}, f$, and $g$ be continuous. Then the pair of integral equations $(16,17)$ has a unique, continuous solution $U, W$ for $t \geq 0$, and $U(0)=h(0-), W(0)=h(0+)$. Hence the problem $(11,15)$ also has a solution.

Corollary 1. Assume additionally that $u_{0}, w_{0}, f$, and $g$ are continuously differentiable with $f(0)=g(0)=0$, and that $h$ is twice continuously differentiable with $h^{\prime}(0)=0$. Then $U^{\prime}, W^{\prime}$ exist and are continuous on $t \geq 0$, and $U^{\prime}(0)=c_{u}^{2} h^{\prime \prime}(0), W^{\prime}(0)=c_{w}^{2} h^{\prime \prime}(0)$. 
Proof. We proceed by formally differentiating (16) with respect to time. For the first integral in (16) we have, using the substitution $t-\sigma=\tau$,

$$
\begin{aligned}
\frac{d}{d t} \int_{0}^{t} G(1, t ; 1, \tau) U(\tau) d \tau=\frac{d}{d t} \int_{0}^{t} G(1, t ; 1, t-\sigma) U(t-\sigma) d \sigma \\
=G(1, t ; 1,0) U(0)+\int_{0}^{t} G(1, t ; 1, t-\sigma) U^{\prime}(t-\sigma) d \sigma \\
\quad=2^{-1}(\pi t)^{-1 / 2}\left[1-e^{-1 / c^{2} t}\right] U(0)+\int_{0}^{t} G(1, t ; 1, \tau) U^{\prime}(\tau) d \tau,
\end{aligned}
$$

because $G(1, t ; 1, t-\sigma)$ is independent of $t$. All except the final integral in (16) are differentiated formally in this manner. The last integral can be rewritten in the form (18); in this representation the second integral is clearly differentiable, with the derivative vanishing as $t \rightarrow 0+$. In the first integral we introduce the change of variable $\xi=2 t^{1 / 2} \theta$ and integrate once by parts to get

$$
\begin{aligned}
& \frac{d}{d t}(\pi t)^{-1 / 2} \int_{0}^{t} e^{-\xi^{2} / 4 c w^{2} t} h(\xi) d \xi \\
= & \frac{d}{d t} 2 \pi^{-1 / 2} \int_{0}^{1 / 2 t^{1 / 2}} e^{-\theta 2 / c w^{2}} h\left(2 t^{1 / 2} \theta\right) d \theta \\
= & -2^{-1} \pi^{-1 / 2} t^{-3 / 2} e^{-1 / 4 c w^{2} t} h(1) \\
& +2 \pi^{-1 / 2} \int_{0}^{1 / 2 t 1 / 2} e^{-\theta 2 / c w^{2}} h^{\prime}\left(2 t^{1 / 2} \theta\right) t^{-1 / 2} \theta d \theta \\
= & -2^{-1} \pi^{-1 / 2} t^{-3 / 2} e^{-1 / 4 c w^{2} t} h(1) \\
& +2 c_{w}^{2}(\pi t)^{-1 / 2}\left[h^{\prime}(0)-e^{-1 / 4 c w^{2} t} h^{\prime}(1)\right] \\
& +c_{w}^{2}(\pi t)^{-1 / 2} \int_{0}^{1} e^{-\xi^{2} / 4 c w^{2} t} h^{\prime \prime}(\xi) d \xi .
\end{aligned}
$$

The final integral is the solution at $x=0$ of the Cauchy problem $v_{t}=c_{w}^{2} v_{x x}$ with initial temperature distribution $2 c_{w}^{2} h^{\prime \prime}(x)(0 \leq x \leq 1)$ and zero elsewhere; as such it is continuous for $t \geq 0$ and has limit $c_{w}^{2} h^{\prime \prime}(0)$ as $t \rightarrow 0$.

We finally get for $W^{\prime}$ the integral equation

$$
\begin{aligned}
W^{\prime}(t)= & \frac{K}{k_{w}} \int_{0}^{t} G(1, t ; 1, \tau)\left[U^{\prime}(\tau)-W^{\prime}(\tau)\right] d \tau-2 \int_{0}^{t} G_{\xi}(1, t ; 1, \tau) W^{\prime}(\tau) d \tau \\
& -c_{w}^{-2} \pi^{-1 / 2} t^{-3 / 2} e^{-1 / c^{2} t} h(0)-\frac{2}{k_{w}} \int_{0}^{t} G(1, t ; 1, \tau) g^{\prime}(\tau) d \tau \\
& +c_{w}^{-2} \pi^{-1 / 2} t^{-3 / 2} e^{-1 / 4 c w^{2 t}} w_{0}(0)+2 \int_{0}^{t} G_{\xi}(1, t ; 0, \tau) w_{0}^{\prime}(\tau) d \tau \\
& -2^{-1} \pi^{-1 / 2} t^{-3 / 2} e^{-1 / 4 c w^{2 t}} h(1)-2 c_{w}^{2}(\pi t)^{-1 / 2} e^{-1 / 4 c w^{2} t} h^{\prime}(1) \\
& +2 c_{w}^{2}(\pi t)^{-1 / 2} \int_{0}^{1} e^{-\xi^{2} / 4 c w^{2 t}} h^{\prime \prime}(\xi) d \xi
\end{aligned}
$$




$$
-\frac{d}{d t} \int_{0}^{1}(\pi t)^{-1 / 2} e^{-(2-\xi)^{2} / 4 \omega^{2} t} h(\xi) d \xi ;
$$

there is a similar integral equation for $U^{\prime}$. Since $z^{\alpha} e^{-z} \rightarrow 0$ as $z \rightarrow+\infty$, the inhomogeneous terms are continuous on $t \geq 0$; thus these integral equations are of the form (19, 20). Let $\bar{U}, \bar{W}$ be their unique solution. By integrating and reversing the steps above, we find that $U(0)+\int_{0}^{t} \bar{U}(\sigma) d \sigma, W(0)+\int_{0}^{t} \bar{W}(\sigma) d \sigma$ solve the integral equations $(16,17)$. Therefore $U(t)=U(0)+\int_{0}^{t} \bar{U}(\sigma) d \sigma, W(t)=W(0)+\int_{0}^{t} \bar{W}(\sigma) d \sigma$, and $U^{\prime}=\bar{U}, W^{\prime}=\bar{W}$ follows.

It is easy to see that $U^{\prime}(0)=c_{u}^{2} h^{\prime \prime}(0), W^{\prime}(0)=c_{w}^{2} h^{\prime \prime}(0)$.

Corollary 2. Assume that $f \equiv g \equiv 0$, that $u_{0}, w_{0} \in C^{2}$, and that $h \in C^{4}$ with $h^{\prime}(0)=h^{\prime \prime}(0)=h^{\prime \prime \prime}(0)=0$. Then $U^{\prime \prime}, W^{\prime \prime}$ are continuous on $t \geq 0$.

The proof is similar to that of the first corollary.

IV. Asymptotic analysis. Set

$$
\begin{gathered}
\hat{u}=\sum_{i=0}^{1} \varepsilon^{i} u^{i}(y, t)+U(y, t, \varepsilon), \quad \hat{v}=\sum_{i=0}^{1} \varepsilon^{i} v^{i}(y, t)+V(y, t, \varepsilon), \\
\hat{w}=\sum_{i=0}^{1} \varepsilon^{i} w^{i}(y, t)+W(y, t, \varepsilon),
\end{gathered}
$$

in (5) to get

$$
\begin{array}{cc}
\sum_{i=0}^{1} \varepsilon^{i}\left[u_{t}^{i}-c_{u}^{2} u_{y y}^{i}\right]+\left[U_{t}-c_{u}^{2} U_{y y}\right]=0 & (-2 \leq y \leq-1), \\
\left.\left(\sum_{i=0}^{1} \varepsilon^{i} u^{i}+U\right)\right|_{t=0}=h(y+1-\varepsilon) & (-2 \leq y \leq-1), \\
\varepsilon v_{t}^{0}+\varepsilon^{2} v_{t}^{1}+\varepsilon V_{t}=K v_{y y}^{0}+\varepsilon K v^{1} y y+K V_{y y} & (-1<y<1), \\
\left.\left(\sum_{i=0}^{1} \varepsilon^{i} v^{i}+V\right)\right|_{t=0}=h(\varepsilon y) & (-1<y<1), \\
\sum_{i=0}^{1} \varepsilon^{i}\left[w_{t}^{i}-c_{w}^{2} w_{y y}^{i}\right]+\left[W_{t}-c_{w}^{2} W_{y y}\right]=0 & (1 \leq y \leq 2), \\
\left.\left(\sum_{i=0}^{1} \varepsilon^{i} w^{i}+W\right)\right|_{t=0}=h(y-1+\varepsilon) & (1 \leq y \leq 2), \\
\left.\left(\sum_{i=0}^{1} \varepsilon^{i} u^{i}+U\right)\right|_{y=-2}=u_{0}(t),\left.\quad\left(\sum_{i=0}^{1} \varepsilon^{i} w^{i}+W\right)\right|_{y=2}=w_{0}(t), \\
\left.\left(\sum_{i=0}^{1} \varepsilon^{i} u^{i}+U\right)\right|_{y=-1}=\left.\left(\sum_{i=0}^{1} \varepsilon^{i} v^{i}+V\right)\right|_{y=-1}, \\
\left.\left(\sum_{i=0}^{1} \varepsilon^{i} v^{i}+V\right)\right|_{y=1}=\left.\left(\sum_{i=0}^{1} \varepsilon^{i} w^{i}+W\right)\right|_{y=1},
\end{array}
$$




$$
\begin{gathered}
\left.k_{u}\left(\sum_{i=0}^{1} \varepsilon^{i} u_{y}^{i}+U_{y}\right)\right|_{y=-1}=\left.K\left(\sum_{i=0}^{1} \varepsilon^{i} v_{y}^{i}+V_{y}\right)\right|_{y=-1}, \\
\left.K\left(\sum_{i=0}^{1} \varepsilon^{i} v_{y}^{i}+V_{y}\right)\right|_{y=1}=\left.k_{w}\left(\sum_{i=0}^{1} \varepsilon^{i} w_{y}^{i}+W_{y}\right)\right|_{y=1} .
\end{gathered}
$$

The hope is to construct the $u^{i}, v^{i}$, and $w^{i}$ in such a way that the remainders $U$ and $W$ tend to zero as $\varepsilon \rightarrow 0+$; then the zeroth-order terms of the asymptotic representations for $\hat{u}$ and $\hat{w}$, and thus $u$ and $w$, will be correct. The necessity in our proof of constructing terms of order $\varepsilon$ will be commented on below.

In the following formal considerations we treat $U, V$, and $W$ as if they and their derivatives were of order $o(\varepsilon)$. Examining zeroth-order terms, we see that $v^{0}$ must satisfy

$$
\begin{gathered}
v_{y y}^{0}=0 \quad(-1<y<1), \\
u^{0}(-1, t)=v^{0}(-1, t), \quad v^{0}(1, t)=w^{0}(1, t), \\
k_{u} u_{y}^{0}(-1, t)=K v_{y}^{0}(-1, t), \quad K v_{y}^{0}(1, t)=k_{w} w_{y}^{0}(1, t)
\end{gathered}
$$

-the problem (6-7) of the introduction-plus the initial condition

$$
v^{0}(y, 0)=h(0)
$$

Since the solution of $(6)$ is

$$
v^{0}(y, t)=\frac{1}{2}(y+1) w^{0}(1, t)-\frac{1}{2}(y-1) u^{0}(-1, t),
$$

$v^{0}$ is uniquely determined without the initial condition $v^{0}(y, 0)=h(0)$. We shall return to this point shortly.

The zeroth-order terms $u^{0}, w^{0}$ must satisfy

$$
\begin{array}{cl}
u_{t}^{0}=c_{u}^{2} u_{y y}^{0}, \quad u^{0}(y, 0)=h(y+1) & (-2 \leq y \leq-1), \\
u^{0}(-2, t)=u_{0}(t) & (t \geq 0), \\
w_{t}^{0}=c_{w}^{2} w_{y y}^{0}, \quad w^{0}(y, 0)=h(y-1) & (1 \leq y \leq 2), \\
w^{0}(2, t)=w_{0}(t) & (t \geq 0),
\end{array}
$$

as well as the coupling conditions (22). From the initial conditions above it follows that $w^{0}(1,0)=u^{0}(-1,0)=h(0)$, which in turn implies that $v^{0}(y, 0)=h(0)$, so the initial condition on $v^{0}$ is indeed satisfied.

From $(22,23)$ we get that

$$
k_{u} u_{y}^{0}(-1, t)=k_{w} w_{y}^{0}(1, t)=\frac{1}{2} K\left[w^{0}(1, t)-u^{0}(-1, t)\right] \quad(t \geq 0)
$$

(24-25) together completely determine $u^{0}, w^{0}$ without reference to the temperature distribution $v^{0}$ in the thin sheet. Returning to the original $x, t$ variables produces (9) for the reduced problem. In order to guarantee that a suitably differentiable solution of the reduced problem exists, we assume until the final paragraph that $h^{\prime}(0)=h^{\prime \prime}(0)=h^{\prime \prime \prime}(0)=0$. By Corollary 2 to Theorem 1 , these conditions guarantee that $u^{0}(-1, t)$ and $w^{0}(1, t)$ are twice continuously differentiable for $t \geq 0$. This in turn means that $v^{0}(y, t) \in C^{2}$.

Ignoring for the moment the initial condition for $v^{1}$, we find from equality of first- 
order terms that $u^{1}, v^{1}, w^{1}$ satisfy the system

$$
\begin{gathered}
\begin{array}{r}
u_{t}^{1}=c_{u}^{2} u_{x x}^{1}, \quad u^{1}(y, 0)=\lim _{\varepsilon \rightarrow 0} \frac{h(y+1-\varepsilon)-h(y+1)}{\varepsilon} \\
=-h^{\prime}(y+1)=0 \quad(-2 \leq y \leq-1), \\
w_{t}^{1}=c_{w}^{2} w_{x x}^{1}, \quad w^{1}(y, 0)=\lim _{\varepsilon \rightarrow 0} \frac{h(y-1+\varepsilon)-h(y-1)}{\varepsilon} \\
=h^{\prime}(y-1)=0 \quad(1 \leq y \leq 2), \\
u^{1}(-2, t)=0, \quad w^{1}(2, t)=0 \quad(t \geq 0), \\
K v_{y y}^{1}=v_{t}^{0} \quad(-1<y<1), \quad(t \geq 0), \\
u^{1}(-1, t)=v^{1}(-1, t), \quad v^{1}(1, t)=w^{1}(1, t) \quad(t \geq 0) .
\end{array}
\end{gathered}
$$

The following explicit formula for $v^{1}$ is an easy consequence of (29-30):

$$
\begin{aligned}
v^{1}(y, t)= & \frac{1}{12 K}\left\{w_{t}^{0}(1, t)(y+1)^{3}-u_{t}^{0}(-1, t)(y-1)^{3}\right\} \\
& +\frac{1}{2}\left\{w^{1}(1, t)(y+1)-u^{1}(-1, t)(y-1)\right\} \\
& -\frac{1}{3 K}\left\{w_{t}^{0}(1, t)(y+1)-u_{t}^{0}(-1, t)(y-1)\right\} .
\end{aligned}
$$

From this it follows readily that $u^{1}, w^{1}$ are solutions (existence proved in Sec. 3) of the system composed of (26-28) and

$$
\begin{aligned}
k_{u} u_{y}^{1}(-1, t)+\frac{1}{2} K\left\{u^{1}(-1, t)-w^{1}(1, t)\right\} & =-\frac{2}{3} u_{t}^{0}(-1, t)-\frac{1}{3} w_{t}^{0}(1, t), \\
k_{w} w_{y}^{1}(1, t)+\frac{1}{2} K\left\{u^{1}(-1, t)-w^{1}(1, t)\right\} & =\frac{2}{3} w_{t}^{0}(1, t)+\frac{1}{3} u_{t}^{0}(-1, t) .
\end{aligned}
$$

Letting $\bar{u}^{1}(x, t)=u^{1}(y, t), \bar{w}^{1}(x, t)=w^{1}(y, t)$, etc., we find on returning to the original variables that the matching conditions (32) become

$$
\begin{aligned}
k_{u} \bar{u}_{x}^{1}(0, t)+\frac{1}{2} K\left\{\bar{u}^{-1}(0, t)-\bar{w}^{1}(0, t)\right\} & =-\frac{2}{3} \bar{u}_{t}^{0}(0, t)-\frac{1}{3} \bar{w}_{t}^{0}(0, t), \\
k_{w} \bar{w}_{x}^{1}(0, t)+\frac{1}{2} K\left\{\bar{u}^{1}(0, t)-\bar{w}^{1}(0, t)\right\} & =\frac{2}{3} \bar{w}_{t}^{0}(0, t)+\frac{1}{3} \bar{u}_{t}^{0}(0, t) .
\end{aligned}
$$

Once $\bar{u}^{0}$ and $\bar{w}^{0}$ are determined, these are matching conditions of the same form as those imposed on $\vec{u}^{0}, \vec{v}^{0}$ except that they are inhomogeneous. By Corollary 1 to Theorem 1 , the right-hand sides of (33) vanish at $t=0$; that they are differentiable has already been seen. Existence of $\bar{u}^{1}, \bar{w}^{1}$ is a consequence of Theorem 1 ; continuous differentiability of $\bar{u}^{1}(0, t)$, $\bar{w}^{1}(0, t)$ and thus of $u^{1}(-1, t), w^{1}(1, t)$ follows from the observation above and Corollary 1 . The continuous differentiability of $u^{1}(-1, t), w^{1}(1, t)$ and of $u_{t}^{0}(-1, t), w_{t}^{0}(1, t)$ guarantees in turn that $v^{1}(y, t)$ is continuously differentiable on $[-1,1] \times[0, \infty)$.

Examination of those terms of (21) that are left shows that the remainders $U, V, W$ must satisfy

$$
\begin{aligned}
U_{t} & =c_{u}^{2} U_{y y}, \quad U(y, 0)=h(y+1-\varepsilon)-h(y+1) & & (-2 \leq y \leq 1), \\
\varepsilon V_{t} & =K V_{y y}-\varepsilon^{2} v_{t}^{1}=K V_{y y}+O\left(\varepsilon^{2}\right) & & (-1<y<1),
\end{aligned}
$$




$$
\begin{aligned}
& V(y, 0)=h(\varepsilon y)-h(0)-\varepsilon v^{1}(y, 0) \\
& (-1<y<1) \text {, } \\
& W_{t}=c_{w}^{2} W_{y y}, \quad W(y, 0)=h(y-1+\varepsilon)-h(y-1) \quad(1 \leq y \leq 2), \\
& U(-2, t)=0, \quad W(2, t)=0 \\
& (t \geq 0), \\
& U(-1, t)=V(-1, t), \quad V(1, t)=W(1, t) \\
& (t \geq 0) \text {, } \\
& k_{u} U_{y}(-1, t)=K V_{y}(-1, t), \quad K V_{y}(1, t)=k_{w} W_{y}(1, t) \quad(t \geq 0) .
\end{aligned}
$$

Our goal is to show that $U$ and $W$ tend to zero as $\varepsilon \rightarrow 0$. Utilizing the Taylor expansion of $h$, we find that (34) and (37) reduce to

$$
\begin{array}{lll}
U_{t}=c_{u}^{2} U_{y y}, & U(y, 0)=O(\varepsilon) & (-2 \leq y \leq-1), \\
W_{t}=c_{w}^{2} W_{y y}, & W(y, 0)=O(\varepsilon) & (1 \leq y \leq 2) .
\end{array}
$$

Let $U^{1}, V^{1}, W^{1}$ satisfy $(34,37-40)$ and

$$
\varepsilon V_{t}^{1}=K V_{y y}^{1}, \quad V^{1}(y, 0)=0 .
$$

By Lemma 2, $\left(34^{\prime}\right)$, and $\left(37^{\prime}\right)$ it follows that $U^{1}=O(\varepsilon), W^{1}=O(\varepsilon)$. Let $U^{2}, V^{2}, W^{2}$ satisfy $(35,38-40)$ and

$$
\begin{gathered}
U_{t}^{2}=c_{u}^{2} U_{y y}^{2}, \quad U^{2}(y, 0)=0, \\
W_{t}^{2}=c_{w}^{2} W_{y y}^{2}, \quad W^{2}(y, 0)=0, \quad V^{2}(y, 0)=0 ;
\end{gathered}
$$

then $U^{2}=O(\varepsilon), W^{2}=O(\varepsilon)$ by Lemma 3. Terms through order $\varepsilon$ were constructed in order that the final term on the right-hand side of (35) should be $O\left(\varepsilon^{2}\right)$.

Consider the right-hand side of (36). Again using the Taylor expansion of $h$, this reduces to

$$
V(y, 0)=-\varepsilon v^{1}(y, 0)+O\left(\varepsilon^{2}\right)=O(\varepsilon) .
$$

Let $U^{3}, V^{3}, W^{3}$ satisfy $(36,38-40)$ and

$$
\begin{gathered}
U_{t}^{3}=c_{u}^{2} U_{y y}^{3}, \quad U^{3}(y, 0)=0, \quad \varepsilon V_{t}^{3}=K V_{y y}, \\
W_{t}^{3}=c_{w}^{2} W_{y y}^{3}, \quad W^{3}(y, 0)=0 .
\end{gathered}
$$

Lemma 2 guarantees that $U^{3}, W^{3}=O(\varepsilon)$. Since $U=U^{1}+U^{2}+U^{3}, W=W^{1}+$ $W^{2}+W^{3}$, we have shown that $U=O(\varepsilon), W=O(\varepsilon)$.

So far we have been assuming that $h^{\prime}(0)=h^{\prime \prime}(0)=h^{\prime \prime \prime}(0)=0$. We now remove these restrictions. Let $h \in C^{2}$, and let $\left\{h_{n}(x)\right\}$ be a sequence of $C^{4}$ functions with the properties that $h_{n}^{(j)}(0)=0(j=1,2,3)$ and $\left|h_{n}(x)-h(x)\right|<1 / n$; it is clear that such sequences exist. Let $u, v, w$ satisfy problem (1-3) and let $u^{n}, v^{n}, w^{n}$ satisfy problem (1-3) with $h_{n}$ in place of $h$. Let $U, W$ and $U^{n}, W^{n}$ be the solutions of the corresponding reduced problems (9). We have shown above that $\left|u^{n}-U^{n}\right|+\left|w^{n}-W^{n}\right|=O(\varepsilon)$. Now a straightforward application of the maximum principle (Lemma 1) to $u-u^{n}-1 / n, v-v^{n}-1 / n, w-w^{n}-1 / n$, and to $u^{n}-u-1 / n, v^{n}-v-1 / n$ shows that $\left|u-u^{n}\right|+\left|w-w^{n}\right| \leq 2 / n$. A similar application of Lemma 4 to $U-U^{n}-1 / n, W-W^{n}-1 / n$ and to $U^{n}-U-1 / n, W^{n}-W-1 / n$ shows that $\left|U-U^{n}\right|+\left|W-W^{n}\right| \leq 2 / n$. It now follows from the triangle inequality that $|u-U|+|w-W| \rightarrow 0$ as $\varepsilon \rightarrow 0$ uniformly in $t$ bounded and $x$. The following theorem summarizes our results.

THEOREM 2. Let the data $u_{0}, w_{0}$, and $h$ be twice continuously differentiable. Then the 
solution $u^{0}(x, t), w^{0}(x, t)$ of problem (9) is an approximation to the solution of (1-3) as $\varepsilon \rightarrow 0$ in the sense that

$$
\begin{array}{ll}
u(x-\varepsilon, t) \rightarrow u^{0}(x, t) & (x \in[-1,0]), \\
w(x+\varepsilon, t) \rightarrow w^{0}(x, t) & (x \in[0,1])
\end{array}
$$

as $\varepsilon \rightarrow 0+$, uniformly in $x$ and $t$ bounded.

\section{REFERENCES}

[1] L. E. Bobisud, Asymptotics of heat conduction in a bar capped with a thin, poorly conductive skin, to appear.

[2] M. H. Protter and H. L. Weinberger, Maximum principles in differential equations, Prentice-Hall, Englewood Cliffs, N.J., 1967

[3] L. I. Rubinstein, The Stefan problem, American Mathematical Society, Providence, R. I., 1971 